\title{
Density of States of Landau Levels in Two-Dimensional Systems from Activated Transport, Magnetocapacitance and Gate Current Experiments
}

\author{
D. Weiss* and K. v. Klitzing \\ Max-Planck-Institut für Festkörperforschung, \\ D-7000 Stuttgart 80, Fed. Rep. of Germany
}

In this publication we demonstrate that a combination of capacitance and gate current experiments together with an analysis of thermally activated conductivity seems to be useful for the determination of the density of states (DOS) of Landau levels in two-dinensional systems. The experimental results suggest a Landau level width not far away from the predictions of the self-consistent Born approximation ( $\mathrm{SCBA}$ ) if the Fermi level is close to the center of a Landau level. The DOS between Landau levels however cannot be explained with such a narrow linewidth and the experiments suygest the existence of a background DOS or an increased linewidth broadening for integer filling factors.

\section{Introduction}

A microscopic theory of the quanturn nall effect should give a correct ciescription not only of the quantized resistivity values $\rho x y=h / i e^{2}$ but also of the transitions between the plateaus and the values of the finite resistivity $\rho_{x x}$. Such transport calculations are extremely complicated since the theory itself is complicated and in adaition not enough information is available about the scattering centers. The published theories are based on certain approximations and assumptions about the cistribution, the strenyth and the range of the scattering potential. A first test whether such assumptions are realistic should be available from a comparison between the calculated and the measured density of states $D(t)$ since calculations of $D(E)$ are much edsier than a transport theory for $\rho_{x x}(B)$ which includes complicated phenomena like localization and correlation. une of the first theories of the density of states (DUS) assumed short-ranye scatterers which leads within the self-consistent Born approximation (SCBÁ) to a broadening of the discrete energy spectrum (expected for an ideal two-aimensional electron gas without scattering).into an elliptic lineshape for the UUS [1]. migher order approximations show that an exionentially decayiny Dus is expected for energies $E-E_{n}$ larger than the linewidth of the Landau levels $E_{n}[2]$, so that a real energy gap with vanishing DOS may not be present but the DOS at midpoint between two Landau levels should decrease drastically if the magnetic field (energy separation between adjacent Landau levels) is increased. Experimental information about the DUS can be obtained from measurements of the specific heat [3], from magnetization ineasurements [4], from temperature-dependent resistivity measurements in the reyine of the Hall plateaus [5], from maynetocapacitance measurements $[6,7]$ or from yate current measurements [8]. In this article we compare the results we have obtained from an analysis of the thermally activated resistivity, maynetocapacitance and gate current measurements carried out on one and the same sample. The following discussion is based on a picture which does not include many-body effects. The notation "density of states (DUS)" in this paper is used to characterize the electronic properties within a sinyle particle picture. All experiments described in the followiny have been carried out on AlGaAs-GaAs heterostructures. 
The temperature dependence of $\min _{x x}$ (where $\rho_{x x}$ means the minimum in the resistivity which corresponds to a Fermi level position very close to the midpoint between two Landau levels) in the temperature ranye $2 K<T<20 \mathrm{~K}$ is usually dominated by an exponential term corresponding to

$$
\min _{x x} \sim \exp \left\{-\frac{E_{a, \max }}{k T}\right\}
$$

where $E_{a, \max }$ denotes the measured activation energy. Measured activation energies $E_{a, m a x}$ for different samples at different magnetic field values are shown in Fig.1. The filling factor $i$, defined as $i=n_{s} \cdot \frac{h}{e b}$ corresponds always to a fully occupied lowest Landau level ( $i=4$ for $(100)$ silicon MOSFETs and $i=2$ for GaAs-AIGaAs heterostructures). Since the measured activation energy $E_{a}$ max agrees fairly well with half of the cyclotron energy $\hbar_{\omega c}$, this activation energy is interureted as the energy difference between the Fermi energy $t_{F}$ and the center of the Landau level $t_{n}$. For the sake of simplicity we assume that the mobility edge of the Landau level is located at the center of the Landau level, in agreement with calculations of the localization length [9] and percolation theories [10]. Furthermore the mobility edge is assumed to remain fixed, independent of the temuerature and the carrier density. Changiny the position of a Lanuau level $t_{n}$ relative to the Fermi energy $E_{F}$ (by changing the maynetic field) results in a reduced activation energy $E_{a}=\left|E_{n}-E_{F}\right|$. This motion of the Landau levels relative to the Fermi level if the filliny factor of the Lanciau levels is varied is clearly visible in Fig.2. A chanye of the filling factor corresponds to a shift of the Ferini level, equivalent to a chanye $\Delta^{n}$ in the carrier density at fixed magnetic field. Measuring now the activation energy as a function of the maynetic field allows us to cieduce a mean value for the DUS:

$$
D(E) \approx \frac{\Delta n}{\Delta t},
$$

where $\Delta E$ is the energy difference between activation energies determined at consecutive magnetic field values. This analysing technique is restricted to the tails of Landau levels and has been described in more detail in a previous publication [8]. Figure 3 shows the reconstructed uos obtained from sample $1\left(n_{\mathrm{S}}=2.60 .1011 \mathrm{~cm}^{-2}, \mu=158,000 \mathrm{~cm} 2 / \mathrm{Vs}\right)$.

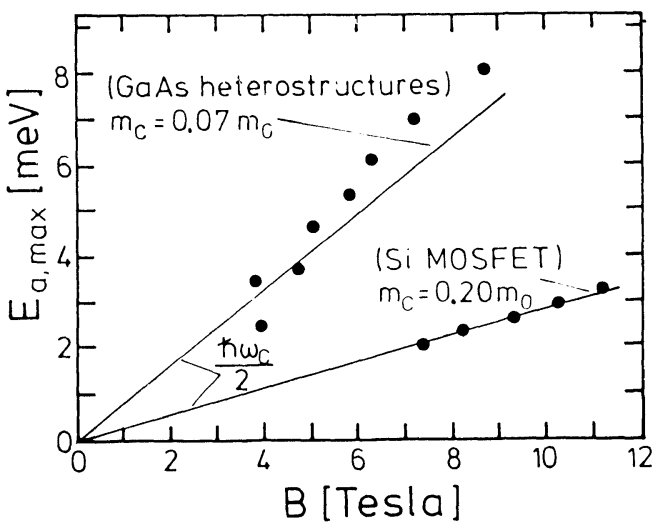

58
Fig. 1:

Measured activation eneryies $E_{\text {a }}$ wax in the resistivity at a fiplifing factor corresuondiny to a fully occuried lowest Lanciau level as a function of the maynetic field $B$. The solid lines correspond to half of the cyclotron eneryy. 


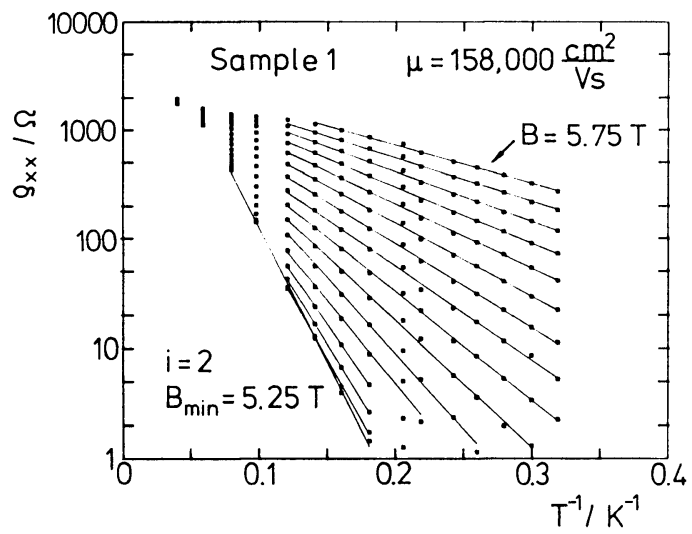

Fig. 2:

Temperature dependence of the resistivity $\rho_{x x}$ at different magnetic fields close to a filling factor $i=2$.
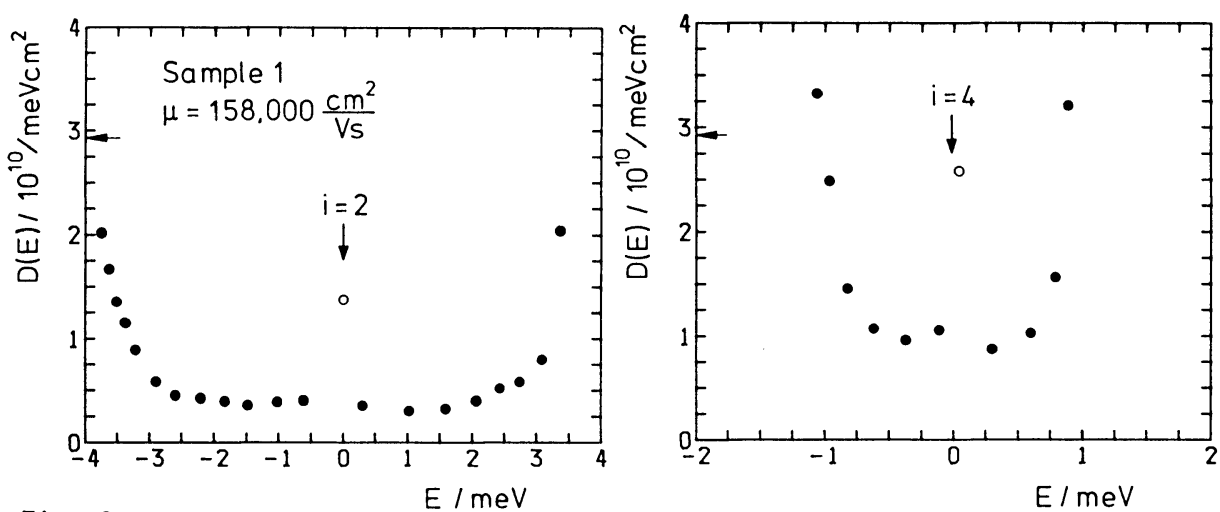

Fig. 3:

Reconstructed DOS for filling factors close to $i=2$ and $i=4$. The arrow marks the zero magnetic field DOS. The energy scale is taken relative to the midpoint between two Landau levels

The DOS between Landau levels does not vanish but shows a value depending on the mobility of the sample and on the magnetic field. Decreasing mobility and decreasing magnetic field results in an increased DOS between Landau levels. The magnetic field dependence of the Dos between Landau levels - obtained from an analysis at filling factors close to $i=2$ and $i=4$ - is contrary to our previous statements. The high Dús close to $t=0$ (Fig. 3 ) is an artefact of the analysis since for a Fermi energy at $t=0$ two Landau levels contribute to $\rho_{x x}$. The reconstruction of the Dús from activated resistivity measurements is restricted to the tails of the Landau levels as mentioned above. Information about the DUS for a Fermi level position close to the center of a Landau level can be obtained from magnetocapacitance measurements described in the next chapter.

\section{Magnetocapacitance}

The capacitance experiments were carried out on gated GaAs-AlGaAs heterostructures with a Hall geometry. The mobilities of the samples described here are between 83,000 and $480,000 \mathrm{~cm}^{2} / \mathrm{Vs}$ for carrier densities in the 
range between $2.27 .10^{11} \mathrm{~cm}^{-2}$ and $2.90 .10^{11} \mathrm{~cm}^{-2}$. For capacitance measurements all the potential probes were short-circuited and acted as a channel contact.

The signal was obtained by measuring phase sensitive the voltage drop between the sample and a high-precision reference capacitor C ref (see Fig. $4 b)$. The signal $V_{\text {meas }}$ is proportional to the capacitance difference $C$ sample ${ }^{-C}$ ref as long as the channel resistance $k_{c h}$ is small compared to the

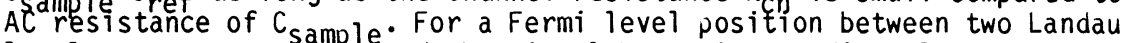
levels $\sigma_{x x}$ goes to zero and the signal $V_{\text {meas }}$ is not directly proportional to the capacitance of the system but is influenced by the low conductivity state of the channel.

The capacitance of a system consisting of a metal-insulator-(with ionized impurities) semiconductor-sandwich (e.g. Au-AlGaAs-GaAs-heterostructure) depends not only on the thickness of the insulator but also on the DOS at the semiconductor side and on parameters of the material. Fig.4a shows the band diagram of a heterostructure including a schottky gate in contact with the AlGaAs. If the two depletion layers interuenetrate each other the total capacitance at a given magnetic field can be expressed as $[8,11]$ :

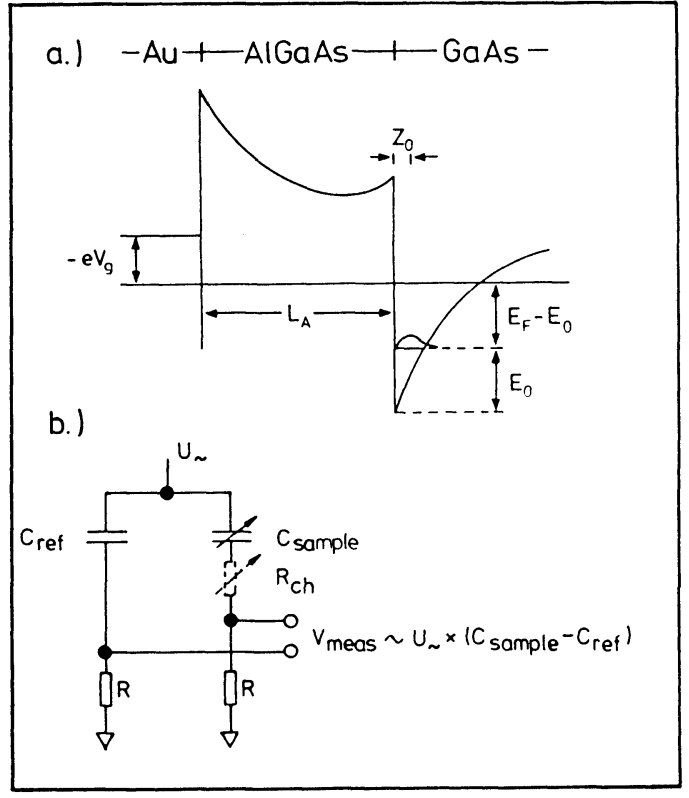

$$
\frac{1}{\tau}=\frac{1}{\tau_{A}}+\frac{\gamma z_{0}}{\varepsilon_{S}}+\left.\frac{1}{e^{2} \frac{d n_{S}}{d\left(E_{F}-E_{0}\right)}}\right|_{E_{F}},
$$

\section{Fig. 4:}

Schematic diagrams of the conduction band edge for a gated GaAsAlGaAs heterostructure showing the quantities used in the derivations (a) and the experimental set up (b). $U$ is the AC component of the applied voltage with an amplitude of about $7 \mathrm{mV}$ and a frequency of $223 \mathrm{~Hz}$

where $C_{A}$ is the capacitance of the insulating AlGaAs layer, $\varepsilon_{S}$ is the dielectric constant of GaAs, $z_{Q}$ is the average position of the electrons in the channel, $\gamma$ is a constant numerical factor between 0.5 and 0.7 , and $\mathrm{dn}_{\mathrm{S}} / \mathrm{d}\left(\mathrm{E}_{\mathrm{F}}-\mathrm{E}_{0}\right)$ is the thermodynamic DOS at the Fermi level, in the following denoted as $\mathrm{dn}_{\mathrm{s}} / \mathrm{dE}_{\mathrm{F}}$. The first two terms on the right-hand side of (3) are 
assumed to be constant in a magnetic field, and thus changes of the capacitance are directly related to changes in the thermodynamic Dus of the $2 D E G$. At $T=0$ the total inverse capacitance in a magnetic field can be expressed as

$$
\frac{1}{C}=\frac{1}{C_{0}}-\frac{1}{e^{2} D_{0}}+\frac{1}{e^{2 D}} \text {, }
$$

where $C_{0}$ denotes the value of the total capacitance at $B=0, D$ is the DUS at the Fermi level in the presence of a magnetic field and $D_{0}$ is the DOS within the lowest subband, equal to $2.9 \times 1010 \mathrm{~cm}-2 \mathrm{meV}-1$ in the absence of a magnetic field. At finite temperatures $D$ has to be replaced by $\mathrm{dn}_{\mathrm{S}} / \mathrm{dt}_{F}$.

The experimental results were compared with calculations of $C(B)$ assuming a Gaussian-1ike Dus of the form:

$$
D(E)=\frac{e}{\pi \hbar} \cdot \frac{1}{\sqrt{2 \pi}} \cdot \frac{B}{\Gamma} \cdot \sum_{n} \exp \left\{-\frac{\left(t-\left(n+\frac{1}{2}\right) \hbar_{\omega_{C}}\right)^{2}}{2 \Gamma^{2}}\right\},
$$

where $\Gamma$ is the broadening parameter of the Gaussian distribution. First the position of the Fermi level $L_{F}$ is determined by solving numerically the equation:

$$
n_{S}=\int_{-\infty}^{\infty} D(E) f\left(t-t_{F}\right) d t
$$

where $f\left(E-E_{F}\right)$ is the Fermi distribution function. The carrier density $n_{S}$ is assumed to be independent of magnetic field and temperature.

In the next step the thermodynamic DuS

$$
\left.\frac{d n_{S}}{d t_{F}}\right|_{E_{F}}=\left.\int_{-\infty}^{\infty} D(E) \cdot \frac{d f\left(t-t_{F}\right)}{d E_{F}}\right|_{E_{F}} d t
$$

is calculated numerically. With the temperature-dependent form of (4) and (7) one obtains $C(B)$. Spin splitting which is sinall compared to the cyclotron energy for GaAs is neglected in the calculations.

Fig. 5 shows the capacitance data (for the same sample as discussed in Fig. 3) at different temperatures together with a theoretical curve calculated on the basis of a magnetic field independent linewidth $r=0.48 \mathrm{meV}$. We have adjusted the fit to the magnetocapacitance maxima since the observed minima may be falsified - at least at high magnetic fields and low temperatures - by the small channel conductance. This argument cannot be used to explain the reduced depth of the capacitance minima at magnetic fields below 2 Tesla since the phase shift due to the channel resistance is negligibly small. However, inhomoyeneities may explain the experimental aata as shown in Fig. 6 where the change in the capacitance due to a Gaussian distribution of the carrier density $n_{S}$ with a brodeniny parameter $\Delta n_{S}=0.016$ $\mathrm{n}_{\mathrm{s}}$ is shown. A remarkable reduction of the depth of the cauacitance minima is visible whereas the maxima remain unchanyed.

The influence of inhomogeneities has been considered in a more sophisticated way by Gerhardits and Gudrnundsson [12] in their statistical mociel for inhomogeneities. Their model is based on the assumption of a caussianshaped DOS and the result of the calculations can De described usiny an effective linewidth $\Gamma$ shown in Fig. 7. This effective linewidth oscillates and a maximum is always obtained for a Fermi level position between two 

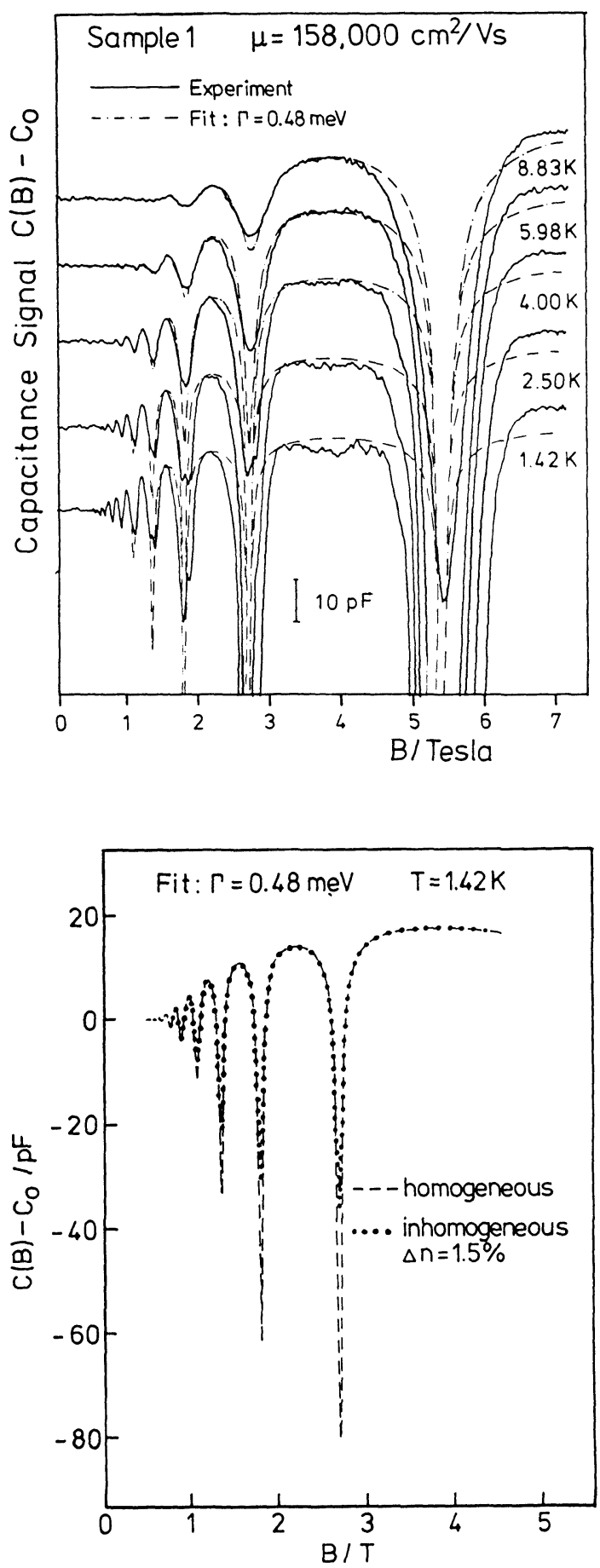

Fig. 5:

Mieasured magnetocapacitance and corresponding fit using a broadening parameter $\Gamma=0.48 \mathrm{meV}$ in the model DUS. For the sake of clarity the curves are shifted vertically
Fig. 6:

Calculated magnetocapacitance showing the influence of inhomoyeneities assuming a Gaussian distribution of the carrier density $n_{S}$ 


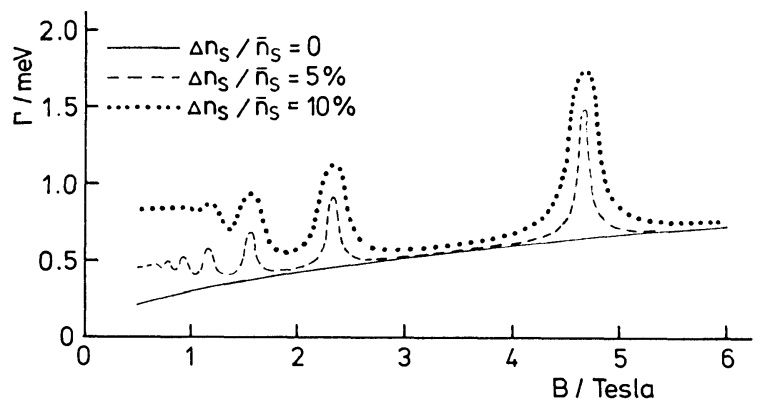

Fig. 7 :

Effective Landau level broadening as a function of B. After [13]

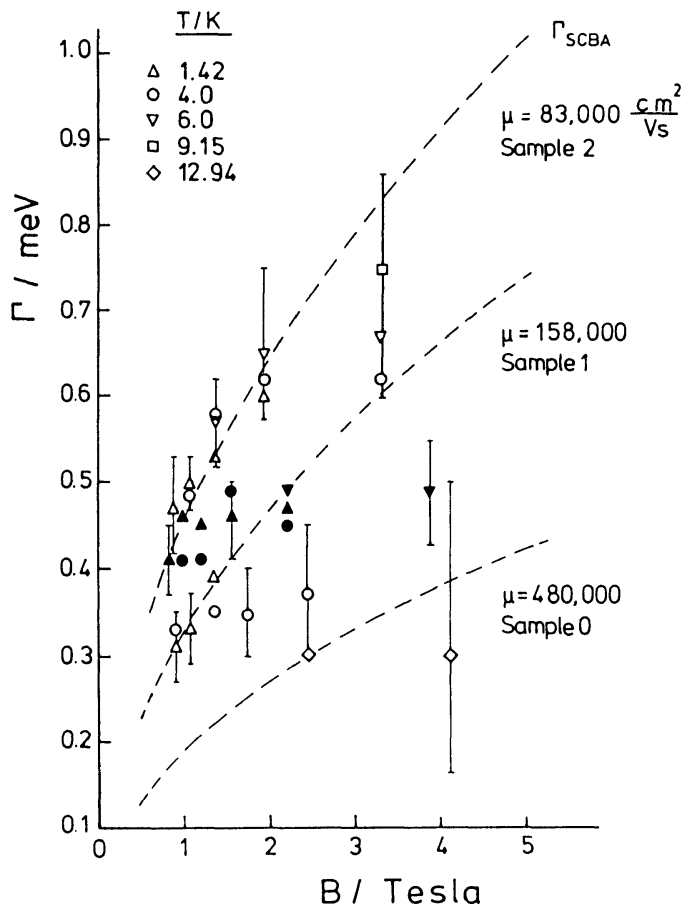

Fig. 8:

Landau level width $\Gamma$ vs. B. Points are obtained by comparing magnetocapacitance maxima with model calculations. The dashed line corresponds to the SCBA- linewidth (8) where $\mu$ is the mobility of the samples. Full symbols correspond to sample 1 and 1 ie in the range between open symbols corresponding to sample 0 and sample 2

Landau levels. This aspect is similar to calculations of the oscillatiny level broadening due to screening effects [14-16].

Figure 8 summarizes the results obtained from an analysis of the magnetocapacitance maxima of three samples. In this figure the broadening parameter $\Gamma$ (see (5)) is plotted as a function of the magnetic field and compared with the 1 inewidth $\Gamma_{S C B A}$, obtained from the selfconsistent Born approximation (SCBA) [1]:

$$
\Gamma_{S C B A}=\frac{e \hbar}{m^{\star}} \sqrt{\frac{2}{\pi} \cdot \frac{B}{\mu}}
$$

where $m^{\star}$ is the effective mass and $\mu$ the mobility of the sample. Fitting the magnetocapacitance maxima means that each point in Fiy. 8 corresuonds 
to a Fermi level position close to the center of a Landau level. The linewidth has been extracted from measurenents at different temperatures incicated by different symbols. Figure 8 shows that the experimentally deduced linewidths are not so far away from the predictions of the SCBA - tine $\sqrt{B}$ dependence of the linewidth $\Gamma$ is more or less visible for the sample with the lowest mobility.

Up to now the model calculation of the magnetocapacitance was based on the assumption that the carrier density in the channel remains constant. This is incorrect, since the difference in the electrochemical potential across the capacitor is fixed and a variation in the capacitance leacis to a charge transfer between the gate electrode and the channel. This small change in the carrier density is unimportant in an analysis of capacitance measurements but is a first order contribution in gate current experiments which will be discussed in the following chapter.

\section{Gate Current}

The assumption that the carrier density $n_{s}$ remains constant changing the magnetic field is not correct. Actually not the carrier density $n_{s}$ but the Fermi level is kept constant during capacitance experiments. Using the notation of Fig.4a this means that the gate voltage $V_{g}$ is kept constant. Varying the magnetic field $B$ then leads to oscillations of the surface potential (bottom of the potential well) and to a charge transfer between gate and channel of the heterostructure. Since the amount of transferred charge is small compared to the two-dimensional carrier density $n_{s}$ the subband edge (taken relative to the bottom of the potential well) is assumed to be constant. The starting point for the model calculations is now no longer (5) but the following equation [8]:

$$
\int_{-\infty}^{\infty} D(E) f\left(E-E_{F}\right) d E+\frac{C_{A}}{e^{2}} E_{F}=\text { const, }
$$

where the constant can be determined at $B=0$. tquation (9) has to be solved numerically to give the correct position of the subband edge relative to the Fermi level and then the magnetocapacitance can be calculated using (7) and the temperature dependent form of (4). Calculating the magnetocaracitance in the way described above results in a broadening of the width of the capacitance minima compared to calculations assuming a constant carrier density $n_{\mathrm{s}}$. The difference however is small and cannot be resolved in Fig. 5. The charge flow mentioned above can be determined by measuring the current between gate and channel as a function of the magnetic field $B$. The current flow is given by

$$
I(B)=A \cdot e \cdot \frac{d n_{S}}{d t}=A \cdot e \frac{d n_{S}}{d B} \cdot \frac{d B}{d t},
$$

where $A$ is the area of the two-dimensional electron yas and $d \dot{b} / d t$ the swee rate of the maynetic field. $\mathrm{dn}_{\mathrm{s}} / \mathrm{dB}$ can be determined by solving (9) at different magnetic fields since the first term on the left-nana side is equal to the carrier density $n_{S}$. The current flow versus maynetic field is shown in Fig. 9. The upper curve shows the experiments (carriea out aydin on sample 1) where a current minimum corresponds to a Dús maximum anu a maximum in the current flow corresponds to a Fermi level position in a minimum of the DUS. The origin of the reverse current peak at about 5.2 Tesla is not clear yet. The experilient is compared with model calculations using a Landau level linewidth (Gaussian) of $\Gamma=0.48 \mathrm{meV}$ (see Fig. 5 and Fig. 8) and $1.35 \mathrm{meV}$. The smaller linewidth $\Gamma=0.48 \mathrm{meV}$ obtained from an 


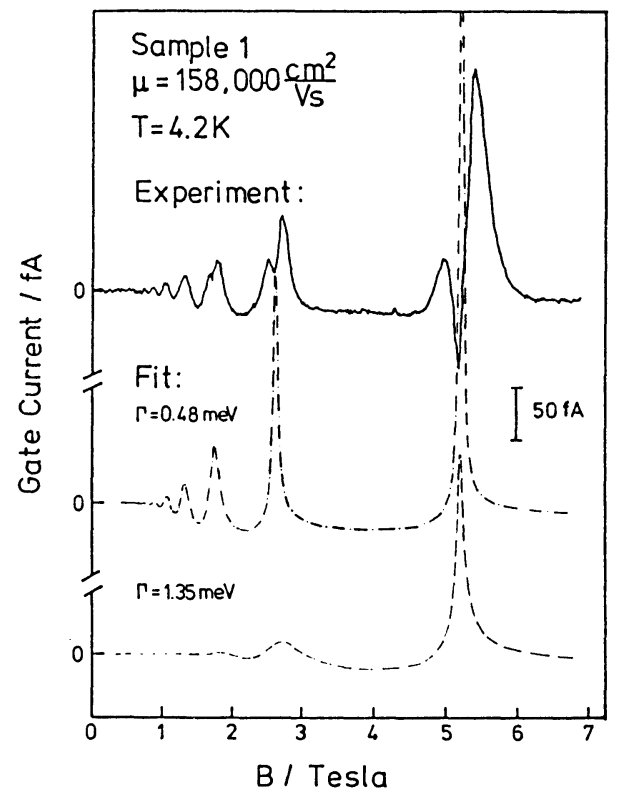

Fig. 9:

Measured and calculated current flow between yate and channel of sample 1 vs. magnetic field

analysis of the magnetocapacitance maxima of the same sample - again describes correctly the experiment for a Fermi level position close to the center of a Landau level. A larger linewidth $\Gamma=1.35 \mathrm{meV}$ cannot fit the oscillations at lower magnetic field but fits approximately the heiyht of the measured current maximum at about 5.4 Tesla (filling factor $i \approx 2$ ).

A more quantitative analysis however is hindered by the reverse current peak. It should be noted that the statistical model $[12,13]$ or a backyround DOS at $i=2$ produces more broadened current maxima, comparable to the experimental ones.

\section{Summary}

Three different experimental methods have been carried out on one and the same sample. These three methods are sensitive to different eneryetical regions of the DOS. An analysis of the thermally activated resistivity restricted to the tails of the Landau levels - shows a nonvanishing Dus between Landau levels depending on mobility and magnetic field which cannot be explained within the SCBA or higher order approxinations. Maynetocapacitance measurements however are mainly restricted to the maxima of the ius.

If the Fermi level position is close to the center of a Landau level the magnetocapacitance data can be explained with a Gaussian-shaped DOS where the linewidth $\Gamma$ follows roughly the SCBA predictions. Gate current experiments in principle are sensitive to maxima as well as to minima in the Dus. For a Fermi level position in a maximum of the DOS the gate current measurements show the same result as magnetocapacitance measurements. The explanation of the gate current measurements for a Fermi level position between two Landau levels requires a background DOS or an increased linewidth broadening. 


\section{Acknowledgement}

We would like like to thank R. Gerhardts and V. Gudmundsson for stimulating discussions and their interest in this work. We are grateful to K. Ploog and $G$. Weimann for providing the samples and we appreciate the cooperation with E. Stahl and V. Mosser at earlier stages of this work.

\section{References}

‡ Present address: Physik-Department $t 16$, Technische Universität München, D-8046 Garching

1. T. Ando, Y. Uemura: J.Phys.Soc.Jap. 36, 959 (1974)

2. R.R. Gerhardts: Surf.Sci. 58, 227 (1976)

3. E. Gornik, R. Lassnig, G. Strasser, H.L. Störmer, A.C. Gossard, W. Wiegmann: Phys.Rev.Lett. 54, 1820 (1986)

4. J.P. Eisenstein, H.L. Störme $\bar{r}$, V. Narayanamurti, A.Y. Cho, A.C. Gossard: Phys.Rev.Lett. 55, 875 (1985)

5. E. Stah 1, D. Weiss, G. Weimann, K. V.Klitzing, K. Ploog: J.Phys. C18, L783 (1985)

6. T.P. Smith, B.B. Goldberg, P.J. Stiles, M. Heiblum: Phys.Kev. B32, 2696 (1985)

7. V. Mosser, D. Weiss, K. V.Klitzing, K. Ploog, G. Weimann: Solid State Commun. 58, 5 (1986)

8. D. Weiss. K. v. Klitzing, V. Mosser: in Two-Dimensional Systems: Physics and New Devices, ed. by G. Bauer, F. Kuchar, H. Heinrich, Springer Ser. Solid State Sci., Vol. 67 (Suringer, Berlin, neidelbery 1986) p. 204

9. T. Ando: J.Phys.Soc.Jap. 53, 31001 (1984)

10. S.A. Trugman: Phys.Rev. B2T, 7539 (1983)

11. F. Stern: Phys.Rev. B5, 4891 (1972)

12. R.R. Gerhardts, V. Gudmundsson: Phys.kev. B34, 2999 (1986)

13. V. Gudmundsson, R.R. Gerharats: to be published

14. R. Lassnig, E. Gornik: Solid State Commun. 47, 959 (1983)

15. T. Ando, Y. Murayama: J.Phys.Soc. Japan. 54, 1519 (1985)

16. W. Cai, T.S. Ting: Phys.Rev. B33, 3967 (1986) 